TITLE:

\title{
Linear viscoelasticity of a single semiflexible polymer with internal friction.
}

\section{$\operatorname{AUTHOR}(S)$ :}

Hiraiwa, Tetsuya; Ohta, Takao

\section{CITATION:}

Hiraiwa, Tetsuya ...[et al]. Linear viscoelasticity of a single semiflexible polymer with internal friction.. The Journal of chemical physics 2010, 133(4): 044907.

ISSUE DATE:

2010-07-30

URL:

http://hdl.handle.net/2433/130006

RIGHT:

(C) 2010 American Institute of Physics 


\title{
Linear viscoelasticity of a single semiflexible polymer with internal friction
}

\author{
Tetsuya Hiraiwa ${ }^{\text {a) }}$ and Takao Ohta ${ }^{\text {b) }}$ \\ Department of Physics, Graduate School of Science, Kyoto University, Kyoto 606-8502, Japan
}

(Received 7 May 2010; accepted 18 June 2010; published online 30 July 2010)

\begin{abstract}
The linear viscoelastic behaviors of single semiflexible chains with internal friction are studied based on the wormlike-chain model. It is shown that the frequency dependence of the complex compliance in the high frequency limit is the same as that of the Voigt model. This asymptotic behavior appears also for the Rouse model with internal friction. We derive the characteristic times for both the high frequency limit and the low frequency limit and compare the results with those obtained by Khatri et al. (C) 2010 American Institute of Physics. [doi:10.1063/1.3463427]
\end{abstract}

\section{INTRODUCTION}

Recent experimental advances in the manipulation of single molecules, such as optical tweezers and atomic force microscopy together with single-molecule fluorescence, ${ }^{1}$ make us accessible to examine mechanical or relaxation properties of single polymer chains in the nanoscale with piconewton sensitivity. ${ }^{2,3}$ Such experiments have provided us with some new kinds of information which is difficult to obtain in bulk experiments. ${ }^{1}$

Under these experimental developments, viscoelastic properties of single polymer chains have been investigated intensively experimentally. ${ }^{4-9}$ Some of these experiments show that internal friction, or additional friction due to the conformational change other than the translational solvent friction, is needed for analyzing the data. ${ }^{8,9}$ It should be noted that, in the present paper, we use the term "internal friction" as the friction due to the dissipation by the conformational change, which does not include the dissipation due to rubbing of each segments.

The Rouse model ${ }^{10}$ is often employed for theoretical studies of polymer dynamics. Khatri et al. investigated the Rouse model with internal friction (RIF model) and derived the analytical expression for the linear viscoelasticity of a single polymer chain. ${ }^{11}$ Quite recently, Deutsch investigated the dynamics of the RIF model in the absence of solvent and derived the autocorrelation function of the conformation. ${ }^{12}$ However, the Rouse model is not applicable when the stiffness of the chain is dominant. Such a situation occurs even for very flexible polymer chains when they are strongly extended. ${ }^{13-16}$ Biological molecules, such as DNA and cytoskelton, are known to have non-negligible stiffness. ${ }^{17,18}$ Therefore, theoretical investigation of the dynamics of the wormlike-chain incorporating the nonlinearity due to the stiffness and the inextensibility is needed. The simplest model which takes account of the stiffness is the wormlikechain (WLC) model, ${ }^{19-22}$ which is a continuous model of a chain with the persistence length $\ell_{p}$ and the finite total length $L$. The analytical studies of this model are very limited compared with those of numerical simulations. ${ }^{23-28}$ In particular,

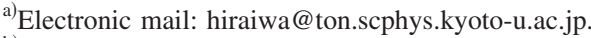

${ }^{b)}$ Electronic mail: takao@scphys.kyoto-u.ac.jp.
}

theoretical studies for the dynamics of the wormlike-chain with internal friction have not been available only with a few exception. $^{9,29}$

Recently, we have developed an analytical method to derive the linear viscoelasticity of a semiflexible polymer chain, ${ }^{15,16}$ which is based on the multiple scale analysis developed by Hallatschek et al $^{30,31}$ for a semiflexible chain without internal friction. Scaling analysis has also been employed to explain the power-law exponents for both the high frequency limit and the low frequency limit. ${ }^{16}$

In the present paper, we shall investigate the complex compliance and complex modulus of a single semiflexible polymer chain with internal friction. We extend the procedure in the previous papers. ${ }^{15,16}$ The method takes account of the nonuniformity of the line tension along the chain ${ }^{30,32}$ and this formulation allows us to deal with the strong nonlinearity due to the inextensibility and the stiffness of the chain.

The outline of the paper is as follows. In Sec. II, we present the wormlike-chain model with internal friction, and, in Sec. III, describe the theory of the linear viscoelasticity. ${ }^{15,16}$ In Sec. IV, we derive the general expression of the complex compliance and complex modulus as well as the characteristic time scale. In Sec. V, we derive the characteristic times associated with the viscoelastic relaxation at the low frequency limit where the finiteness of the chain length plays a crucial role and the scale-separation method breaks down. Summary and discussion are given in Sec. VI. In the Appendix, we discuss the wormlike-chain with friction due to rotation of the segments.

\section{MODEL}

We start with the wormlike-chain model with the generalized bending rigidities

$$
\begin{aligned}
H= & \int_{0}^{L} d s\left(\frac{\kappa_{4}}{2}\left|\frac{\partial^{2} \boldsymbol{r}}{\partial s^{2}}(s, t)\right|^{2}+\frac{\kappa_{6}}{2}\left|\frac{\partial^{3} \boldsymbol{r}}{\partial s^{3}}(s, t)\right|^{2}+\cdots\right. \\
& \left.+\frac{\kappa_{2 n}}{2}\left|\frac{\partial^{n} \boldsymbol{r}}{\partial s^{n}}(s, t)\right|^{2}+f(s, t)\left|\frac{\partial \boldsymbol{r}}{\partial s}(s, t)\right|^{2}\right),
\end{aligned}
$$

where $t$ denotes the time, $s$ is the length along the chain from one end, $L$ is the total length, and $\boldsymbol{r}(s, t)$ represents the con- 
formation of the chain. The last term on the right-hand side (rhs) of Eq. (1) arises from the inextensibility constraint

$$
\left|\frac{\partial \boldsymbol{r}}{\partial s}(s, t)\right|^{2}=1 \text {. }
$$

The Lagrange multiplier $f(s, t)$ can be interpreted as the line tension. ${ }^{30,33}$ In this paper, we introduce the positive bending rigidities at all orders $\kappa_{4}, \kappa_{6}, \cdots$ for generality of the theory. The ordinary wormlike-chain model is given by retaining only the $\kappa_{4}$ term. In order to describe the dynamics, we assume that the chain conformation obeys the following overdamped Langevin equation together with Eq. (1):

$\mathcal{L} \frac{\partial \boldsymbol{r}}{\partial t}(s, t)=-\mathcal{M} \boldsymbol{r}(s, t)+\frac{\partial}{\partial s}\left(f(s, t) \frac{\partial \boldsymbol{r}}{\partial s}(s, t)\right)+\boldsymbol{g}(s, t)+\boldsymbol{\xi}(s, t)$,

where $\mathcal{L}$ is the friction coefficient tensor operator given by

$$
\mathcal{L}=\zeta+\zeta_{4} \frac{\partial^{4}}{\partial s^{4}}+\cdots+(-1)^{n} \zeta_{2 n} \frac{\partial^{2 n}}{\partial s^{2 n}} .
$$

The other operator $\mathcal{M}$ is defined as

$$
\mathcal{M}=\kappa_{4} \frac{\partial^{4}}{\partial s^{4}}-\kappa_{6} \frac{\partial^{6}}{\partial s^{6}}+\cdots+(-1)^{n} \kappa_{2 n} \frac{\partial^{2 n}}{\partial s^{2 n}} .
$$

The coefficients $\zeta$ and $\zeta_{n}(n=2,4,6, \cdots)$ are defined as tensors. The tensor $\zeta$ means the friction coefficient between polymer and solvents. The term $-\zeta_{2} \partial^{2} \boldsymbol{r} / \partial s^{2}$ represents the dissipation induced by rotation of the tangent vector and hence should not be considered as an "internal" friction. We shall discuss the effect of this term separately in the Appendix. The simplest nontrivial case,

$$
\mathcal{L}=\zeta+\zeta_{4} \frac{\partial^{4}}{\partial s^{4}},
$$

has been studied by Poirier et ll $^{29}$ for the internal conformation rearrangement of the filament with a finite radius. Khatri et $a l^{9}{ }^{9}$ have employed this kind of wormlike-chain model to derive the characteristic relaxation time which is in good agreement with the experimental data. High order friction coefficients $\zeta_{6}, \zeta_{8}, \cdots$ are also introduced to study those effects from a general point of view. The third term $\boldsymbol{g}(s, t)$ on the rhs of Eq. (3) is the external force and the fourth term $\boldsymbol{\xi}(s, t)$ is the random force which has zero-average and satisfies the fluctuation-dissipation relation of the second kind

$$
\begin{aligned}
& \left\langle\xi_{i}(s, t)\right\rangle=0, \\
& \left\langle\xi_{i}(s, t) \xi_{j}\left(s^{\prime}, t^{\prime}\right)\right\rangle=2 k_{B} T \mathcal{L}_{i j} \delta\left(s-s^{\prime}\right) \delta\left(t-t^{\prime}\right) \delta_{i j} .
\end{aligned}
$$

The friction coefficient tensor $\zeta$ is divided into the transverse and the longitudinal parts as

$$
\zeta=\zeta_{\perp}\left(1-\frac{\partial \boldsymbol{r}}{\partial s} \otimes \frac{\partial \boldsymbol{r}}{\partial s}\right)+\zeta_{\|} \frac{\partial \boldsymbol{r}}{\partial s} \otimes \frac{\partial \boldsymbol{r}}{\partial s} .
$$

The tensor $\zeta_{n}$ is also divided similarly. All the scalar coefficients $\zeta_{\|}, \zeta_{\perp}, \zeta_{\|, n}$, and $\zeta_{\perp, n}$ are assumed to be positive.

\section{MULTIPLE SCALE ANALYSIS}

The multiple scale analysis developed by Hallatschek et $a l^{30,31}$ for a semiflexible chain without internal friction can be extended to Eq. (3). Hallatschek et al. have considered the situation that a chain is elongated by an external force $f$ applied at the two ends. The conformation vector $\boldsymbol{r}(s, t)$ is divided into two components. One is parallel to the elongation direction (along the $x$-axis) and the other is perpendicular to it, i.e., $\boldsymbol{r}(s, t)=\left(s-r_{\|}, \boldsymbol{r}_{\perp}\right)$. The basic assumption is the weak bending condition such that $\left(\partial \boldsymbol{r}_{\perp}(s, t) / \partial s\right)^{2}$ $=O(\check{\epsilon}) \ll 1$, where the smallness parameter is introduced as $\check{\epsilon}=k_{B} T /\left(\kappa_{4} f+\kappa_{6} \ell^{2} f+\cdots\right)^{1 / 2}$ with a characteristic length $\ell$. That is, the elastic energy due to the external force is much larger than the thermal energy. We assume, for simplicity, that not only $\check{\epsilon}$ but also $\epsilon \equiv k_{B} T /\left(\kappa_{4} f\right)^{1 / 2} \geq \check{\epsilon}$ is small. In this situation one has $\partial r_{\|} / \partial s=(1 / 2)\left(\partial \boldsymbol{r}_{\perp} / \partial s\right)^{2}+O\left(\check{\epsilon}^{2}\right)$.

The tension propagation equation is derived by means of the multiple scale analysis. ${ }^{30}$ We define the time-integration of the line tension as

$$
F(s, t)=\int_{0}^{t} d \widetilde{t} f(s, \widetilde{t}) .
$$

Equation for $F(s, t)$ is given in terms of the dimensionless quantities by

$$
\begin{aligned}
K \frac{\partial^{2}}{\partial \hat{s}^{2}} \hat{F}(\hat{s}, \hat{t})= & \int_{0}^{\infty} d \hat{q}\left\{\frac{1-\exp (-A(\hat{q}, \hat{s}, \hat{t}))}{c(\hat{q}) \hat{q}^{2}+1}-\frac{2 \hat{q}^{2}}{b(\hat{q})} \int_{0}^{\hat{t}} d \tilde{t}\right. \\
& \times \exp (-A(\hat{q}, \hat{s}, \hat{t})+A(\hat{q}, \hat{s}, \tilde{t}))\},
\end{aligned}
$$

where $K=\pi \zeta_{\perp} / \zeta_{\|}$and

$$
\begin{aligned}
& \hat{F}(\hat{s}, \hat{t})=\frac{\xi^{2}}{\kappa_{4} \tau_{\xi}} F(s, t), \\
& A(\hat{q}, \hat{s}, \hat{t})=\frac{2 \hat{q}^{2}}{b(\hat{q})}\left\{c(\hat{q}) \hat{q}^{2} \hat{t}+\hat{F}(\hat{s}, \hat{t})\right\}
\end{aligned}
$$

with $\hat{q}=\xi q, \hat{s}=\epsilon^{1 / 2} s \xi^{-1}$ and $\hat{t}=t / \tau_{\xi}$. The length scale $\xi$ and the time scale $\tau_{\xi}$ are given, respectively, by

$$
\begin{gathered}
\xi=\left(\frac{\kappa_{4}}{f_{0}}\right)^{1 / 2}, \\
\tau_{\xi}=\frac{\kappa_{4} \zeta_{\perp}}{f_{0}^{2}} .
\end{gathered}
$$

The Fourier transform is defined by

$$
\boldsymbol{r}_{\perp}(s, t)=\int_{-\infty}^{\infty} \frac{d q}{2 \pi} \widetilde{\boldsymbol{r}}_{\perp}(q, t) e^{i q s} .
$$

In Eqs. (11) and (13), the functions $b(\hat{q})$ and $c(\hat{q})$ are defined, respectively, by

$$
\begin{aligned}
& b(\hat{q})=1+r_{4} \hat{q}^{4}+\cdots+r_{2 n} \hat{q}^{2 n}, \\
& c(\hat{q})=1+u_{6} \hat{q}^{2}+\cdots+u_{2 n} \hat{q}^{2 n-4},
\end{aligned}
$$

where 


$$
\begin{aligned}
& r_{2 n} \equiv \frac{\zeta_{\perp, 2 n}}{\zeta_{\perp} \xi^{2 n}} \\
& u_{2 n} \equiv \frac{\kappa_{2 n}}{\kappa_{4} \xi^{2 n-4}} .
\end{aligned}
$$

We derive the linear force-strain relation from Eq. (11). The external forces $f(0, t)$ and $f(L, t)$ at the chain ends are assumed to be given by $f(0, t)=f(L, t)=f_{0}$ for $t<0$ and $f(0, t)=f(L, t)=f_{0}+\Delta f(t)$ for $t>0$ and we put $f(s, t)=f_{0}$ $+\Delta f(s, t)$. The relation between the chain extension and the force $\Delta f(t)$ has been obtained by Hallatschek et al. ${ }^{30,31}$ They have introduced the concept of the stored excess length defined by

$$
\rho(s, t)=\frac{1}{2}\left(\frac{\partial \boldsymbol{r}_{\perp}}{\partial s}\right)^{2} .
$$

Since the parallel component of the end-to-end distance is given by $R_{\|} \equiv L-\left(r_{\|}(L)-r_{\|}(0)\right)$, one obtains the relation

$$
\left\langle\Delta R_{\|}\right\rangle(t)=-\int_{0}^{L}\langle\Delta \rho\rangle(s, t) d s+o(\check{\epsilon}),
$$

where $\Delta$ stands for a deviation from $t=0$ and $\langle\cdots\rangle$ means a statistical average. The deviation $\langle\Delta \rho(s, t)\rangle$ is related with the line tension as

$$
\langle\Delta \bar{\rho}\rangle(s, t)=-\zeta_{\|}^{1} \frac{\partial^{2}}{\partial s^{2}} \Delta F(s, t),
$$

where $\langle\Delta \bar{\rho}(s, t)\rangle$ is the bulk value of $\langle\Delta \rho(s, t)\rangle$ and $\Delta F(s, t)$ $=\int_{0}^{t} d x \Delta f(s, x)$. The relation between $\Delta F(s, t)$ and $\Delta f(t)$ can be determined through the boundary condition in solving Eq. (11). Therefore, from Eqs. (22) and (23), we obtain $\left\langle\Delta R_{\|}\right\rangle(t)$ as a function of $\Delta f(t)$.

Linearizing Eq. (11) with respect to $\Delta \hat{F}(\hat{s}, \hat{t})=\hat{F}(\hat{s}, \hat{t})-\hat{t}$, we obtain

$$
K \frac{\partial^{2}}{\partial \hat{s}^{2}} \widetilde{\Delta F}(\hat{s}, \hat{\omega})-N(i \hat{\omega}) \widetilde{\Delta F}(\hat{s}, \hat{\omega})=0
$$

where the Fourier-Laplace transformation is defined for an arbitrary function $x(t)$ by

$$
\widetilde{x}(\omega)=\int_{0}^{\infty} d t x(t) \exp (-i \omega t) .
$$

The dimensionless frequency $\hat{\omega}$ is given by $\hat{\omega}=\omega \tau_{\xi}$ and

$$
\begin{aligned}
N(i \hat{\omega})= & -4 \int_{0}^{\infty} d y \frac{1}{b(y)}\left\{\frac{y^{4}}{2 y^{2}\left(c(y) y^{2}+1\right)+i \hat{\omega} b(y)}\right. \\
& \left.-\frac{1}{2} \frac{y^{2}}{c(y) y^{2}+1}\right\} .
\end{aligned}
$$

In the high frequency limit $\hat{\omega} \rightarrow \infty$, the function $N(i \hat{\omega})$ becomes

$$
N(i \hat{\omega}) \sim T_{1}+i \frac{T_{2}}{\hat{\omega}},
$$

where

$$
T_{1}=2 \int_{0}^{\infty} d y \frac{1}{b(y)} \frac{y^{2}}{c(y) y^{2}+1},
$$

and

$$
T_{2}=4 \int_{0}^{\infty} d y \frac{y^{4}}{b(y)^{2}} .
$$

This is valid as long as $\boldsymbol{\zeta}_{n} \neq 0$ for $n \geq 4$ so that the integral in Eq. (26) converges. It is noted that the real part of $N(i \hat{\omega})$ tends to a constant $T_{1}$, whereas the imaginary part is inversely proportional to the frequency.

Before closing this section, we mention that the scale separation breaks down for extremely small values of the frequency $\omega \tau_{\mathrm{fs}} \ll 1$ with $\tau_{\mathrm{fs}}=L^{2} f_{0}^{-1} \zeta_{\perp} b(\pi / L)$, because the integral over the wavenumber should be replaced by the summation of the discrete modes due to the finite size effects. ${ }^{16}$ This case will be analyzed in Sec. V.

\section{LINEAR VISCOELASTICITY}

The force-strain relation can be obtained from Eqs. (22)-(24). ${ }^{16}$ We consider the situation that an oscillatory force is applied at the two ends for $t>0$ as

$$
f(0, t)=f(L, t)=f_{0}+f_{A} \sin (\omega t) .
$$

The scaled complex compliance $\hat{J}(\omega)=\hat{J}^{\prime}(\omega)+i \hat{J}^{\prime \prime}(\omega)$ is defined by

$$
\frac{\left\langle\Delta R_{\|}(t)\right\rangle}{L}=\frac{\epsilon}{2 \pi^{2}} \frac{f_{A}}{f_{0}}\left[\hat{J}^{\prime}(\omega) \sin (\omega t)-\hat{J}^{\prime \prime}(\omega) \cos (\omega t)\right] .
$$

Applying the same procedure as the case without internal friction, ${ }^{5,16,34}$ we obtain the complex compliance from Eq. (24) as

$$
\begin{aligned}
& \hat{J}^{\prime}(\omega)=\frac{1}{\omega \tau} \operatorname{Im}\left(\alpha \sqrt{N(i \hat{\omega})} \tanh \left(\frac{\alpha \sqrt{N(i \hat{\omega})}}{2}\right)\right), \\
& \hat{J}^{\prime \prime}(\omega)=\frac{1}{\omega \tau} \operatorname{Re}\left(\alpha \sqrt{N(i \hat{\omega})} \tanh \left(\frac{\alpha \sqrt{N(i \hat{\omega})}}{2}\right)\right),
\end{aligned}
$$

where

$$
\alpha \equiv \frac{\zeta_{\|}^{1 / 2}\left(k_{B} T\right)^{1 / 2} f_{0}^{1 / 4} L}{\pi^{1 / 2} \zeta_{\perp}^{1 / 2} \kappa_{4}^{3 / 4}}=\sqrt{\frac{\zeta_{\|}}{\pi \epsilon \zeta_{\perp}}} \frac{L}{\ell_{p}} .
$$

By using the dimensionless constant $\alpha$, we may introduce another characteristic time $\tau$ as

$$
\tau \equiv \frac{k_{B} T \zeta_{\|} L^{2}}{4 \pi^{2} \kappa_{4}^{1 / 2} f_{0}^{3 / 2}}=\frac{\alpha^{2}}{4 \pi} \tau_{\xi} .
$$

Note that Eqs. (31)-(35) are the same as those for the case without internal friction. ${ }^{15,16}$ Only the functional form of $N(i \hat{\omega})$ is different and contains the information of the internal friction.

For the low frequency regime $\hat{J}^{\prime}$ is independent of $\omega$ and $\hat{J}^{\prime \prime}$ has a power-law dependence:

$$
\hat{J}^{\prime}(\omega) \sim 16 \pi^{3} \int_{0}^{\infty} d \hat{q} \frac{1}{\left(c(\hat{q}) \hat{q}^{2}+1\right)^{2}},
$$




$$
\hat{J}^{\prime \prime}(\omega) \sim \frac{\pi^{2} \kappa_{4}^{1 / 2} \zeta_{\perp}^{1 / 2}}{2 f_{0}} \omega^{1 / 2}
$$

This is the same as the case without internal friction ${ }^{15,16}$ except for $c(\hat{q})$ in Eq. (36). In the high frequency limit we obtain from Eq. (27) as

$$
\begin{aligned}
& \hat{J}^{\prime}(\omega) \sim \frac{T_{2}}{\alpha \tau_{\xi}^{2}}\left\{\frac{\tanh \left(C_{1}\right)}{C_{1}}+\frac{1}{2 \cosh ^{2}\left(C_{1}\right)}\right\} \frac{1}{\omega^{2}}, \\
& \hat{J}^{\prime \prime}(\omega) \sim \frac{4 \pi T_{1}^{1 / 2}}{\alpha \tau_{\xi}} \tanh \left(C_{1}\right) \frac{1}{\omega},
\end{aligned}
$$

with $C_{1}=\alpha T_{1}^{1 / 2} / 2$. It is interesting to see that these $\omega$-dependences are coincident with those of the Voigt model. This fact implies that, in the high frequency limit, the dynamics is governed by the single structural relaxation with the characteristic time $\tau_{V}$ defined through the dimensionless time $\hat{\tau}_{V}=\tau_{V} \kappa_{4} /\left(\zeta_{\perp} L^{4}\right)$ by

$$
\begin{aligned}
\hat{\tau}_{V} & =\frac{\kappa_{4}}{\zeta_{\perp} L^{4}} \times \frac{\hat{J}^{\prime \prime}(\hat{\omega} \rightarrow+\infty)}{\omega \hat{J}^{\prime}(\hat{\omega} \rightarrow+\infty)} \\
& =\frac{2 T_{1}}{T_{2}} \frac{1}{1+\left(\alpha T_{1}\right) / \sinh \left(\alpha T_{1}\right)} \hat{f}_{0}^{-2},
\end{aligned}
$$

where $\hat{f}_{0}=f_{0} L^{2} / \kappa_{4}$. The first equality comes from the fact that the complex compliance of the Voigt-model obeys $\widehat{J_{V}^{\prime \prime}}(\omega) / \widehat{J_{V}^{\prime}}(\omega)=\omega \tau_{V}$.

The complex modulus is related with the complex compliance as

$$
\begin{aligned}
& \hat{G}^{\prime}(\omega)=\frac{\hat{J}^{\prime}(\omega)}{\hat{J}^{\prime}(\omega)^{2}+\hat{J}^{\prime \prime}(\omega)^{2}}, \\
& \hat{G}^{\prime \prime}(\omega)=\frac{\hat{J}^{\prime \prime}(\omega)}{\hat{J}^{\prime}(\omega)^{2}+\hat{J}^{\prime \prime}(\omega)^{2}} .
\end{aligned}
$$

In the high frequency limit, we obtain

$$
\begin{aligned}
& \hat{G}^{\prime}(\omega) \sim \frac{T_{2} \alpha}{T_{1}^{2}}\left\{\frac{C_{1}}{\tanh \left(C_{1}\right)}+\frac{C_{1}^{2}}{\sinh ^{2}\left(C_{1}\right)}\right\}, \\
& \hat{G}^{\prime \prime}(\omega) \sim \frac{\alpha \tau_{\xi}}{4 \pi T_{1}^{1 / 2}} \frac{1}{\tanh \left(C_{1}\right)} \omega .
\end{aligned}
$$

The results given by Eqs. (36)-(44) are valid irrespective of the highest order derivatives with respect to $s$ in $\mathcal{L}$ and $\mathcal{M}$ as long as the fourth derivative exists. The case that there is no fourth derivative, $\mathcal{L}=\zeta-\zeta_{2}\left(\partial^{2} / \partial s^{2}\right)$, will be discussed in the Appendix.

Hereafter, we consider the simplest nontrivial case for the internal friction where the equation of motion is given by

$$
\begin{aligned}
\zeta \frac{\partial \boldsymbol{r}}{\partial t}(s, t)+\boldsymbol{\zeta}_{4} \frac{\partial^{5} \boldsymbol{r}}{\partial t \partial s^{4}}(s, t)= & -\kappa_{4} \frac{\partial^{4} \boldsymbol{r}}{\partial s^{4}}(s, t)+\frac{\partial}{\partial s}\left(f(s, t) \frac{\partial \boldsymbol{r}}{\partial s}(s, t)\right) \\
& +\boldsymbol{g}(s, t)+\boldsymbol{\xi}(s, t) .
\end{aligned}
$$

This model has been investigated by Poirier et al. ${ }^{29}$ and Khatri et al. ${ }^{9}$ Poirier et al. have calculated numerically the
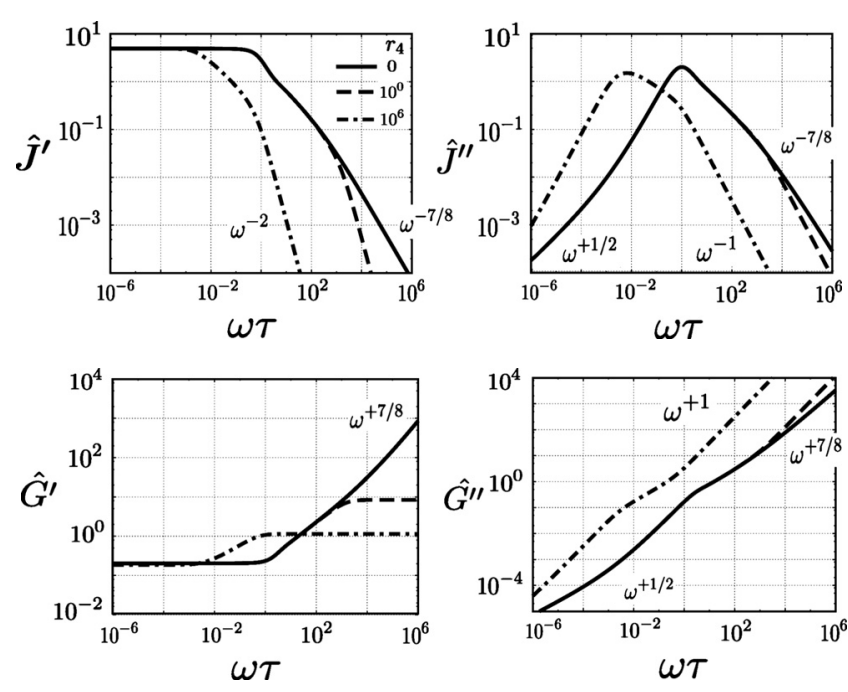

FIG. 1. $\hat{J}^{\prime}, \hat{J}^{\prime \prime}, \hat{G}^{\prime}$, and $\hat{G}^{\prime \prime}$ as a function of $\omega \tau$ for $\alpha=100.0$ and for $r_{4}=0$ (full line), $r_{4}=1$ (broken line), and $r_{4}=10^{6}$ (dotted broken line), where $\tau$ has been defined by Eq. (35).

autocorrelation function of the chain conformation omitting the tension term. ${ }^{29}$ Khatri et al. have evaluated the relaxation time by neglecting the solvent friction and assuming that the system has a single relaxation time as the Voigt-model. ${ }^{9} \mathrm{We}$ apply our general theory to derive the linear viscoelastic behavior analytically.

In the system governed by Eq. (45), the functions $b(\hat{q})$ and $c(\hat{q})$ are given by $1+r_{4} \hat{q}^{4}$ and 1 , respectively. The constants $T_{1}$ and $T_{2}$ defined by Eqs. (28) and (29), respectively, are readily evaluated as

$$
\begin{aligned}
& T_{1}\left(r_{4}\right)=\frac{\pi\left[\sqrt{2} r_{4}^{1 / 4}-2+\sqrt{2} r_{4}^{-1 / 4}\right]}{2\left(1+r_{4}\right)}, \\
& T_{2}\left(r_{4}\right)=\frac{\pi}{2 \sqrt{2}} r_{4}^{-5 / 4} .
\end{aligned}
$$

Note that $T_{1}$ and $T_{2}$ diverge in the limit $r_{4} \rightarrow 0$. When the internal friction is absent, i.e., $r_{4}=0$, the high frequency behavior of $N(i \hat{\omega})$ is given by $N(i \hat{\omega}) \sim 2 S_{1} \hat{\omega}^{1 / 4}+i 4 S_{2} \hat{\omega}^{1 / 4}$ with $S_{1}=\int_{0}^{\infty} d q\left(4 q^{8}+1\right) \quad$ and $S_{2}=\int_{0}^{\infty} d q q^{4} /\left(4 q^{8}+1\right) .^{15,16} \quad$ This asymptotic behavior is entirely different from that of Eq. (27) and hence $T_{1}$ and $T_{2}$ are singular in the limit $r_{4} \rightarrow 0$. The compliance and the modulus for several values of $r_{4}$ are plotted in Fig. 1 for $\alpha=100$ and in Fig. 2 for $\alpha=1$.

It should be emphasized that from Eqs. (46) and (47) and the relation $r_{4}=\hat{\zeta}_{\perp, 4} \hat{f}_{0}$, the $\hat{f}_{0}$-dependence of the dimensionless relaxation time $\hat{\tau}_{V}$ given by Eq. (40) is characterized only by a dimensionless parameter $\hat{\zeta}_{\perp, 4} \equiv \zeta_{\perp, 4} /\left(\zeta_{\perp} L^{4}\right)$. The asymptotic form of the relaxation time is given by

$$
\hat{\tau}_{V}=4 \hat{\zeta}_{\perp, 4}
$$

for $r_{4} \rightarrow 0$ whereas it is given by 

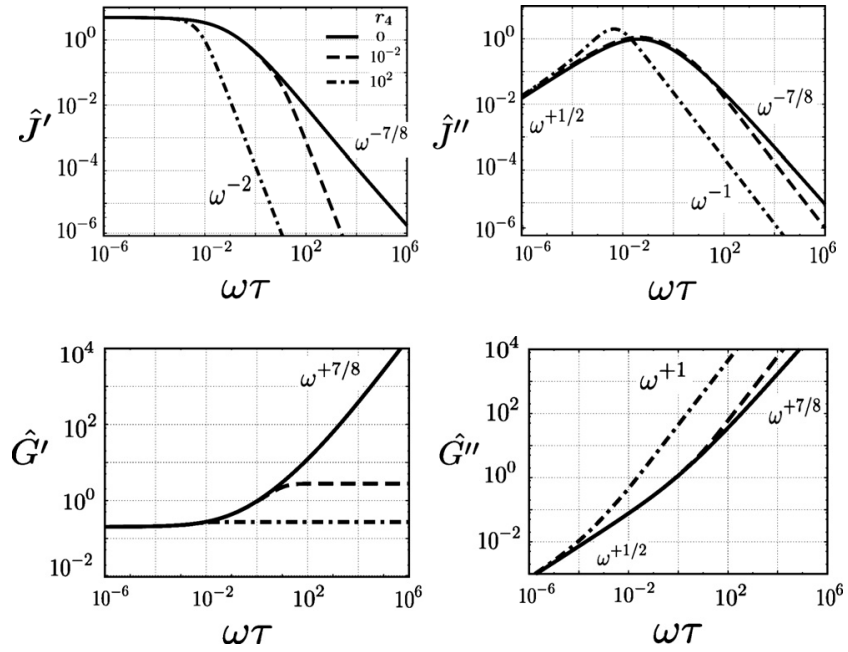

FIG. 2. $\hat{J}^{\prime}, \hat{J}^{\prime \prime}, \hat{G}^{\prime}$, and $\hat{G}^{\prime \prime}$ as a function of $\omega \tau$ for $\alpha=1.0$ and for $r_{4}=0$ (full line), $r_{4}=10^{-2}$ (broken line) and $r_{4}=10^{2}$ (dotted broken line), where $\tau$ has been defined by Eq. (35).

$$
\hat{\tau}_{V}=\frac{2 \hat{\zeta}_{\perp, 4}^{1 / 2}}{\hat{f}_{0}}
$$

for $r_{4} \rightarrow \infty$. Note that Eq. (48) does not contain the background constant force $\hat{f}_{0}$.

\section{LOW FREQUENCY LIMIT AND CHARACTERISTIC TIME}

In this section, we present the linear response for the extremely small frequency regime $\omega \tau_{\mathrm{fs}} \ll 1\left[\tau_{\mathrm{fs}}\right.$ has been defined below Eq. (29)], in which we cannot employ the multiple scale analysis given by Eq. (11) since all the modes enter equally. In this situation, we may approximate the line tension to be uniform along the chain, i.e.,

$$
f(s, t)=f(t)=f_{0}+f_{A} \sin (\omega t) .
$$

From the transverse part of Eq. (3) together with Eq. (50), the complex compliance for $\omega \tau_{\mathrm{fs}} \ll 1$ is obtained as

$\hat{J}^{\prime}(\omega)=\frac{2 \pi^{2} f_{0}^{3 / 2} \kappa_{4}^{1 / 2}}{L} \sum_{n=1}^{\infty} \frac{1}{\left[\kappa_{4}(n \pi / L)^{2} c(\xi n \pi / L)+f_{0}\right]^{2}}$,

$$
\hat{J}^{\prime \prime}(\omega)=\frac{2 \pi^{2} f_{0}^{3 / 2} \kappa_{4}^{1 / 2}}{L} \sum_{n=1}^{\infty} \frac{L^{2}}{2 \pi^{2} n^{2}} \frac{\zeta_{\perp} b(\xi n \pi / L)}{\left[\kappa_{4}(n \pi / L)^{2} c(\xi n \pi / L)+f_{0}\right]^{3}} \omega .
$$

These are valid for both the clamped-end case and the freeend case.

Therefore, the characteristic time for the corresponding Voigt model is given from Eqs. (45), (51), and (52) in terms of the scaled variables by

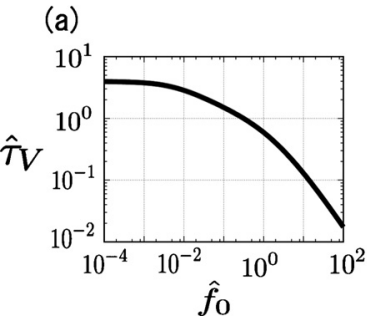

(b)

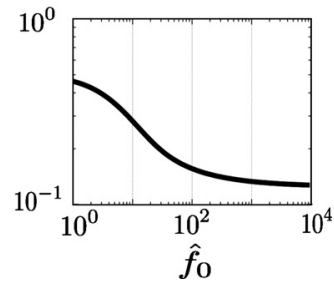

FIG. 3. Dimensionless characteristic time $\hat{\tau}_{V}$ as a function of $\hat{f}_{0}$ for (a) the high frequency limit and (b) the low frequency limit with $\hat{\zeta}_{\perp, 4}=1.0$.

$$
\hat{\tau}_{V}=\left\{\sum_{n=1}^{\infty} \frac{1}{2 \pi^{2} n^{2}} \frac{1+\hat{\zeta}_{\perp, 4}(n \pi)^{4}}{\left[(n \pi)^{2}+\hat{f}_{0}\right]^{3}}\right\}\left\{\sum_{n=1}^{\infty} \frac{1}{\left[(n \pi)^{2}+\hat{f}_{0}\right]^{2}}\right\}^{-1},
$$

where $\hat{\tau}_{V}=\tau_{V} \kappa_{4} /\left(\zeta_{\perp} L^{4}\right), \hat{f}_{0}=f_{0} L^{2} / \kappa_{4}$, and $\hat{\zeta}_{\perp, 4}=\zeta_{\perp, 4} /\left(\zeta_{\perp} L^{4}\right)$. Figure 3 displays the $\hat{f}_{0}$-dependence of $\hat{\tau}_{V}$. Figure $3($ a) shows $\hat{\tau}_{V}$ in the high frequency limit obtained from Eqs. (40), (46), and (47), whereas $\hat{\tau}_{V}$ in the low frequency limit given by Eq. (53) is shown in Fig. 3(b). We examine the $f_{0}$-dependence of $\tau_{V}$ in the low frequency limit in further detail. In the limit of the large external force, $\hat{f}_{0} / \pi^{2} \gg 1$ and $\pi^{2} \hat{f}_{0}^{3 / 2} \hat{\zeta}_{\perp, 4} / 8 \gg 1$, we may replace $\Sigma_{n=1}^{\infty}$ by $\int_{0}^{\infty} d \hat{q} \hat{f}_{0}^{1 / 2} / \pi$ and approximate $1+\hat{\zeta}_{\perp, 4}(n \pi)^{4}$ by $\hat{\zeta}_{\perp, 4}(n \pi)^{4}$ in Eq. (53) so that $\hat{\tau}_{V}$ is evaluated analytically as

$$
\hat{\tau}_{V}=\frac{\hat{\zeta}_{\perp, 4}}{8}
$$

This clearly indicates that $\tau_{V}$ is independent of $\hat{f}_{0}$ in the high frequency limit. Note that the factor $1 / 8$ is consistent with the numerical results in Fig. 3(b) for large values of $\hat{f}_{0}$. Khatri et $a l .{ }^{9}$ have obtained a similar result but with the numerical factor $1 / 32$. On the other hand, $\hat{\tau}_{V}$ for the high frequency given by Eq. (49) is inversely proportional to $\hat{f}_{0}$ for $r_{4} \gg 1$, i.e., for large values of $\hat{\zeta}_{\perp, 4}$ and $\hat{f}_{0}$.

\section{SUMMARY AND DISCUSSION}

We have studied the linear viscoelastic behavior of a single semiflexible chain with internal friction by applying the multiple scale analysis. ${ }^{30,31}$ By internal friction, we mean the friction associated with conformational change of chain. It is found that the asymptotic behavior in the high frequency limit coincides with that of Voigt model as Eqs. (38) and (39) as long as $n \geq 4$. We have investigated the simplest case ${ }^{9,29}$ governed by Eq. (45) in detail and derived the relaxation times $\tau_{V}$ both in the high frequency limit and in the extremely low frequency regime. It is found that $\tau_{V}$ in the high frequency limit is inversely proportional to the prestretching force $f_{0}$ for $f_{0} \rightarrow \infty$ as Eq. (49), whereas $\tau_{V}$ in the extremely low frequency regime is independent of $f_{0}$ for $f_{0} \rightarrow \infty$ as Eq. (54).

The scale separation is justified when the characteristic length parallel to the chain $\ell_{\|}$is much larger than the characteristic length perpendicular to the chain $\ell_{\perp} \cdot{ }^{16,30,34}$ Even when this is not satisfied, the scale separation occurs in the 
high frequency limit in the absence of internal friction ${ }^{16}$ since there is a scaling property such that $\ell_{\|} \sim \omega^{-1 / 8}$ and $\ell_{\perp}$ $\sim \omega^{-1 / 4}$. However, when the internal friction is present, this is not the case. Both $\ell_{\|}$and $\ell_{\perp}$ do not diverge but take a finite value in the high frequency limit because of the strong energy dissipation at the small length scale due to the internal friction.

The Voigt-like power law dependence in the high frequency limit, $J^{\prime} \propto \omega^{-2}$ and $J^{\prime \prime} \propto \omega^{-1}$, also appears in the Rouse model with internal friction (RIF model). ${ }^{11}$ The reason can be understood as follows. When the internal friction is absent, the characteristic wavenumber for chain deformations increases indefinitely as the frequency of the external force is increased. On the other hand, when the internal friction is present, the effect of the internal friction is stronger for shorter wavelength and hence the characteristic wavenumber is not divergent. This intuitive argument can be confirmed by the following semiquantitative explanation. First of all, let us consider the RIF model given by the following equation:

$$
\zeta \frac{\partial \boldsymbol{r}(n, t)}{\partial t}=k \frac{\partial^{2} \boldsymbol{r}(n, t)}{\partial n^{2}}+\zeta_{i} \frac{\partial}{\partial t} \frac{\partial^{2} \boldsymbol{r}(n, t)}{\partial n^{2}}+\boldsymbol{\xi}(n, t),
$$

where $\zeta$ is the friction coefficient between polymer and solvents, $\zeta_{i}$ is the internal friction constant, $k$ is the spring constant between the neighboring pairs, $\boldsymbol{r}(n, t)$ is the position of the $n$th monomer, and $\boldsymbol{\xi}(n, t)$ is the random force acting on the $n$th monomer. ${ }^{11}$ One notes the relation $q$ $\sim \sqrt{\zeta \omega /\left(k+\zeta_{i} \omega\right)}$ comparing the left-hand side and the first and second terms of the right-hand side of Eq. (55), where $q$ and $\omega$ are the characteristic wavenumber and frequency, respectively. It is evident that $q$ does not diverge for $\omega \rightarrow \infty$ as long as $\zeta_{i}$ exists. For the WLC model, the analysis is not so simple because Eq. (3) has a strong nonlinearity. Nevertheless, we note that the characteristic wavenumber $q=\xi^{-1} y$, or $\ell_{\perp}^{-1}$, in Eqs. (26)-(29) is not divergent for $\omega \rightarrow \infty$. This is quite different from the fact that $\ell_{\perp}^{-1} \sim \omega^{1 / 2} \rightarrow \infty$ when the internal friction is absent. ${ }^{16}$

At present, there are few experiments which can be compared with the theoretical results obtained in this paper. Khatri et $a l .{ }^{9}$ have carried out experiments of strain-stress response of a biopolymer by atomic force microscopy. They have found that the characteristic relaxation time $\tau_{K}$ is independent of the prestretching force $f_{0}$. In fact, their data indicate that $\tau_{K} \approx 0.03[\mathrm{~ms}]$ for $30<f_{0}<200[\mathrm{pN}]$. Here, we examine whether or not this is consistent with our theoretical result shown in Eq. (53), which should be valid in the extremely low frequency regime $\omega \tau_{\mathrm{fs}} \ll 1$. If we identify $\tau_{K}$ with $\tau_{V}$ and use the relation (54) and the experimental data $\kappa_{4} \sim 1.0\left[\mathrm{pN} \mathrm{nm}^{2}\right]$, we have $\zeta_{\perp, 4} \sim 0.24\left[\mathrm{pN} \mathrm{ms} \mathrm{nm}{ }^{2}\right]$. Using the values for unfolded polypeptides I27 in their experiment, $\quad f_{0} \sim 100[\mathrm{pN}], \quad L \sim 140[\mathrm{~nm}], \quad \zeta_{\perp} \sim 1.9$ $\times 10^{-5}\left[\mathrm{pN} \mathrm{ms} \mathrm{nm}^{-2}\right]$ and $\xi \sim 0.1[\mathrm{~nm}]$, we obtain $\tau_{\mathrm{fs}}$ $=L^{2} f_{0}^{-1} \zeta_{\perp} b(\pi \xi / L) \approx 4[\mu \mathrm{s}]$. Since the frequency of oscillation of the strain is $\omega \sim 10[\mathrm{kHz}]$ in the experiments, ${ }^{9}$ the condition $\omega \tau_{\mathrm{fs}} \ll 1$ is indeed satisfied. It is also noted that other characteristic quantities are obtained as $\zeta_{\perp} L^{4} / \kappa_{4} \sim 7.3$ $\times 10^{3}[\mathrm{~ms}], \kappa_{4} / L^{2} \sim 5.1 \times 10^{-5}[\mathrm{pN}]$ and $\hat{\zeta}_{\perp, 4} \sim 3.2 \times 10^{-5}$, and we can confirm the condition for asymptotic behavior
(54) by $\hat{f}_{0} / \pi^{2} \sim 2.0 \times 10^{5} \gg 1$ and $\pi^{2} \hat{f}_{0}^{3 / 2} \hat{\zeta}_{\perp, 4} / 8 \sim 1.1 \times 10^{5}$ $\gg 1$. Although $\hat{\zeta}_{\perp, 4}=1.0$ in Fig. 3(b) is quite different from the experimentally estimated value $\hat{\zeta}_{\perp, 4} \approx 10^{-5}$, this does not cause any inconsistency in the above comparison with experiments since $\hat{f}_{0}$ is sufficiently large both in Fig. 3(b) and in the experiments and the asymptotic relation Eq. (54) holds.

The frequency range of the above experiments of strainstress response has been restricted to the extremely small window. It is desired that experiments by changing the frequency of the external force will be capable so that more quantitative and precise comparison can be made with the theoretical results shown in Figs. 1 and 2, and Eqs. (51) and (52).

\section{ACKNOWLEDGMENTS}

This work was supported by the Grant-in-Aid for the priority area "Soft Matter Physics" from the Ministry of Education, Culture, Sports, Science and Technology (MEXT) of Japan and The Next Generation of Physics, Spun from Universality and Emergence. We would like to thank M. Kageshima and M. Kawakami for useful discussions.

\section{APPENDIX:}

In this appendix, we consider the following Langevin equation:

$$
\begin{aligned}
\boldsymbol{\zeta} \frac{\partial \boldsymbol{r}}{\partial t}(s, t)-\boldsymbol{\zeta}_{2} \frac{\partial^{3} \boldsymbol{r}}{\partial t \partial s^{2}}(s, t)= & -\kappa_{4} \frac{\partial^{4} \boldsymbol{r}}{\partial s^{4}}(s, t)+\frac{\partial}{\partial s}\left(f(s, t) \frac{\partial \boldsymbol{r}}{\partial s}(s, t)\right) \\
& +\boldsymbol{g}(s, t)+\boldsymbol{\xi}(s, t) .
\end{aligned}
$$

In this case, the function $b(\hat{q})$ is given by $1+r_{2} \hat{q}^{2}$. The high frequency behavior of Eq. (26) is not given by Eq. (27) but by

$$
N(i \hat{\omega}) \sim T_{3}\left(r_{2}\right)+i T_{4}\left(r_{2}\right) \hat{\omega}^{-1 / 2},
$$

where $T_{3}\left(r_{2}\right)=\pi /\left(r_{2}^{1 / 2}+r_{2}\right)$ and $T_{4}\left(r_{2}\right)=\pi r_{2}^{-3 / 2}$. Therefore, the complex compliance is given by

$$
\begin{gathered}
\hat{J}^{\prime}(\omega) \sim \frac{2 \pi T_{4}\left(r_{2}\right)}{\alpha \tau_{\xi}^{3 / 2} T_{3}\left(r_{2}\right)^{1 / 2}}\left\{\tanh \left(\frac{\alpha T_{3}\left(r_{2}\right)^{1 / 2}}{2}\right)\right. \\
\left.+\frac{\alpha T_{3}\left(r_{2}\right)^{1 / 2}}{2 \cosh ^{2}\left(\alpha T_{3}\left(r_{2}\right)^{1 / 2} / 2\right)}\right\} \frac{1}{\omega^{3 / 2}}, \\
\hat{J}^{\prime \prime}(\omega) \sim \frac{4 \pi T_{3}\left(r_{2}\right)^{1 / 2}}{\alpha \tau_{\xi}} \tanh \left(\frac{\alpha T_{3}\left(r_{2}\right)^{1 / 2}}{2}\right) \frac{1}{\omega} .
\end{gathered}
$$

These are different from the frequency dependence of the Voigt model. However, the results (A3) and (A4) are not structurally stable. In fact, if there are terms with higher derivatives like the left hand side of Eq. (3), the asymptotic behavior of the compliance is given by Eqs. (38) and (39).

${ }^{1}$ F. Ritort, J. Phys.: Condens. Matter 18, R531 (2006).

${ }^{2}$ T. Strick, J. Allemand, V. Croquette, and D. Vensimon, Phys. Today 54(10), 46 (2001).

${ }^{3}$ Y. Sakai, T. Ikehara, T. Nishi, K. Nakajima, and M. Hara, Appl. Phys. Lett. 81, 724 (2002). 
${ }^{4}$ M. Kawakami, K. Byrne, B. Khatri, T. C. B. McLeish, S. E. Radford, and D. A. Smith, Langmuir 20, 9299 (2004).

${ }^{5}$ M. Kawakami, K. Byrne, B. Khatri, T. C. B. McLeish, S. E. Radford, and D. A. Smith, Langmuir 21, 4765 (2005).

${ }^{6}$ M. Kawakami, K. Byrne, D. J. Brockwell, S. E. Radford, and D. A. Smith, Biophys. J. 91, L16 (2006).

${ }^{7}$ M. Kageshima, T. Chikamoto, T. Ogawa, Y. Hirata, T. Inoue, Y. Naitoh, Y. J. Li, and Y. Sugawara, Rev. Sci. Instrum. 80, 023705 (2009).

${ }^{8}$ B. S. Khatri, M. Kawakami, K. Byrne, D. A. Smith, and T. C. B. McLeish, Biophys. J. 92, 1825 (2007).

${ }^{9}$ B. S. Khatri, K. Byrne, M. Kawakami, D. J. Brockwell, D. A. Smith, S E. Radford, and T. C. B. McLeish, Faraday Discuss. 139, 35 (2008).

${ }^{10}$ P. E. Rouse, J. Chem. Phys. 21, 1272 (1953).

${ }^{11}$ B. S. Khatri and T. C. B. McLeish, Macromolecules 40, 6770 (2007).

${ }^{12}$ J. M. Deutsch, e-print arXiv:1003.0944v1.

${ }^{13}$ R. G. Winkler, S. Keller, and J. O. Rädler, Phys. Rev. E 73, 041919 (2006).

${ }^{14}$ L. Harnau, R. G. Winkler, and P. J. Reineker, J. Chem. Phys. 104, 6355 (1996).

${ }^{15}$ T. Hiraiwa and T. Ohta, J. Phys. Soc. Jpn. 77, 023001 (2008).

${ }^{16}$ T. Hiraiwa and T. Ohta, Macromolecules 42, 7553 (2009).

${ }^{17}$ K. Yoshikawa, M. Takahashi, V. V. Vasilevskaya, and A. R. Khokhlov, Phys. Rev. Lett. 76, 3029 (1996).
${ }^{18}$ D. Mizuno, C. Tardin, C. F. Schmidt, and F. C. MacKintosh, Science 315, 370 (2007).

${ }^{19}$ N. Saitô, K. Takahashi, and Y. Yunoki, J. Phys. Soc. Jpn. 22, 219 (1967).

${ }^{20}$ J. F. Marko and E. D. Siggia, Macromolecules 28, 8759 (1995).

${ }^{21}$ R. Goldstein and S. A. Langer, Phys. Rev. Lett. 75, 1094 (1995).

${ }^{22}$ T. B. Liverpool, Phys. Rev. E 72, 021805 (2005).

${ }^{23}$ E. J. Hinch, J. Fluid Mech. 271, 219 (1994).

${ }^{24}$ P. S. Grassia, E. J. Hinch, and L. C. Nitsche, J. Fluid Mech. 282, 373 (1995).

${ }^{25}$ P. S. Grassia and E. J. Hinch, J. Fluid Mech. 308, 255 (1996).

${ }^{26}$ M. Pasquali and D. C. Morse, J. Chem. Phys. 116, 1834 (2002).

${ }^{27}$ M. Somasi, B. Khomami, N. J. Woo, J. S. Hur, and E. S. G. Shaqfeh, J. Non-Newtonian Fluid Mech. 108, 227 (2002).

${ }^{28}$ N. Yoshinaga, K. Yoshikawa, and T. Ohta, Eur. Phys. J. E 17, 485 (2005).

${ }^{29}$ M. G. Poirier and J. F. Marko, Phys. Rev. Lett. 88, 228103 (2002).

${ }^{30}$ O. Hallatschek, E. Frey, and K. Kroy, Phys. Rev. E 75, 031905 (2007).

${ }^{31}$ O. Hallatschek, E. Frey, and K. Kroy, Phys. Rev. E 75, 031906 (2007).

${ }^{32}$ V. Shankar, M. Pasquali, and D. C. Morse, J. Rheol. 46, 1111 (2002).

${ }^{33}$ J. M. Deutsch, Science 240, 922 (1988).

${ }^{34}$ B. Obermayer and E. Frey, Phys. Rev. E 80, 040801(R) (2009). 